\title{
Coherent effects in magneto-transport through Zeeman-split levels
}

\author{
S. A. Gurvitz ${ }^{1,2}$, D. Mozyrsky ${ }^{2}$, and G. P. Berman ${ }^{2}$ \\ ${ }^{1}$ Department of Particle Physics, Weizmann Institute of Science, Rehovot 76100, Israel \\ ${ }^{2}$ Theoretical Division and CNLS, Los Alamos National Laboratory, Los Alamos, NM \\ 87545, USA
}

(November 12, 2018)

\begin{abstract}
We study non-equilibrium electronic transport through a quantum dot or an impurity weakly coupled to ferromagnetic leads. Based on the rate equation formalism we derive the noise spectra for the transport current. We show that, due to quantum interference between different spin components of the current, the spectrum develops peaks or dips at frequencies corresponding to the Zeeman splitting in the quantum dot. A detailed analysis of the spectral structure of the current is carried out for noninteracting electrons as well as for the regime of Coulomb blockade.
\end{abstract}




\section{INTRODUCTION}

Resonant transport through a quantum dot (or impurity) has been investigated in numerous publications. Yet no special attention has been paid to quantum interference effects in this process. These effects can generate oscillations in the resonant current through two (or more) levels of an impurity. These oscillations are similar to the well-known quantum interference effects in the two-slit experiment, and, in turn, produce a peak or a dip in the current power spectrum, depending on the relative phase of the two levels carrying the current [1]. This feature can be experimentally observed in time-resolved measurements of transport currents $[2,3]$. It has been previously argued that the quantum interference effect can explain modulation in the tunneling current at the Larmor frequency in scanning tunneling microscope (STM) experiments [4].

In this paper we investigate the interference effects in polarized magneto-transport. Conductance and I-V curves for spin dependent transport through quantum dots has recently been studied in several publications [5-9]. Here we study the time dependent properties of transport currents. In particular we study the effects related to interference between different spin components of the currents.

These effects can be described schematically as follows. Consider the polarized resonant current from the left reservoir (emitter) to the right reservoir (collector) through a single level of a quantum dot (impurity) in the presence of an external magnetic field. This field would split the resonant level of the dot into a Zeeman doublet, Fig. 1. Let us assume that the polarization axis of electrons in the emitter $(\mathbf{n})$ is different from that of the external magnetic field $(\overline{\mathbf{n}})$. Then a spin-polarized electron from the emitter enters into a superposition of the "spin-up" and the "spin-down" states of the Zeeman doublet, Fig. 1. As a result, the electron wave function in the collector has two components corresponding to different energies of the doublet. Yet these components are orthogonal since they correspond to different spin components and therefore cannot interfere. If, however, there is an additional spin-flip process in the transition between the dot and the collector, the two spin components 
interfere in the collector current. Again, this takes place if the polarization in the collector $\left(\mathbf{n}^{\prime}\right)$ is different from that in the quantum $\operatorname{dot}(\overline{\mathbf{n}})$, Fig. 1. Thus the system operates as a two -path interferometer, where the phase difference between the two paths, i.e., through the upper and the lower spin states in the dot, contributes to the dynamical (or spectral) properties of the collector current.

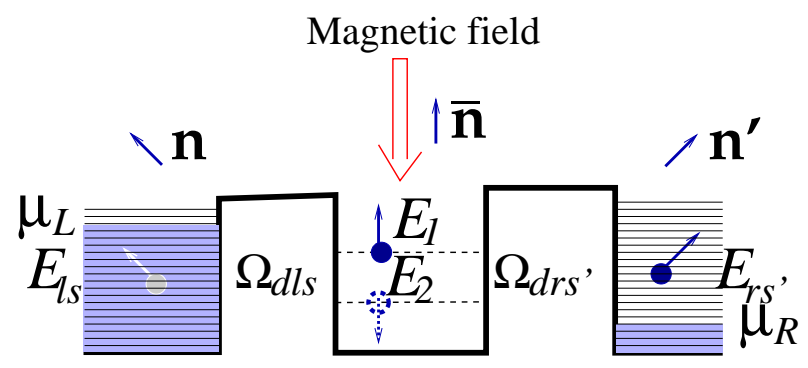

Fig. 1. Resonant tunneling of a polarized electron through a quantum dot. Here the $\Omega$ 's denote the tunneling transition amplitudes between the reservoir states and the Zeeman doublets $\left(E_{1,2}\right)$ of the quantum dot. $\mu_{L, R}$ are the chemical potentials in the left and right reservoirs. The unit vectors $\mathbf{n}, \overline{\mathbf{n}}$ and $\mathbf{n}^{\prime}$ show the polarization axes in the emitter, quantum dot, and the collector, respectively.

These interference effects can be realized experimentally in a heterostructure with a quantum dot sandwiched between the two ferromagnetic leads with easy axes different from those in the dot. A similar set up can be implemented in other systems, such as self-assembled quantum dots [10], ultrasmall particles [11], carbon nanotubes [12] and single molecules [13]. These systems are likely to find prominent technological applications, including random excess memory and magnetic sensors due to giant magnetoresistance effect [14]. The value of Zeeman splitting in the dot is controlled by the external magnetic field. The leads are assumed to be thin magnetic films, so that the magnetic field inside the leads is pinned parallel to the films and therefore the magnetization in the leads remains unaffected by the application of a relatively small external magnetic field. 
The plan of this paper is as follows. In Sect. II we introduce the model and describe in general the rate equation approach for calculations of the resonant current and its noise spectrum. This approach has been obtained directly from the many-body Schrödinger equation describing the entire many-particle system $[15,16]$ and allows one to treat the magnetotransport through quantum dot systems in the most simple and precise way. In Sect. III we consider the case non-interacting (of weakly interacting) electrons. We explain in detail how the rate equations are used to calculate the polarized current and the noise spectrum both for ferromagnetic and non ferromagnetic leads. The results for the time-dependent polarized current are compared with the results of the single-particle model, presented in the Appendix. We explicitly demonstrate how the polarized current exhibits oscillations due to interference effects. In Sect. IV we concentrate on the interacting case. In particular we derive the current spectra in the presence of a Coulomb blockade in the dot. We consider separately the collector and the emitter currents, as well as the circuit current. In Sect. V we summarize our calculations and briefly discuss the potential implications of our results on the noise spectroscopy of quantum dots.

\section{MANY-BODY DESCRIPTION.}

Consider the polarized transport of non-interacting electrons through a quantum dot in the external magnetic field, Fig. 1. The polarization axis of an electron inside the dot $(\overline{\mathbf{n}})$ is different from those in the right and left reservoirs ( $\mathbf{n}$ and $\left.\mathbf{n}^{\prime}\right)$. The tunneling Hamiltonian describing this system can be written as

$$
\begin{aligned}
H=\sum_{l, s} E_{l s} a_{l s}^{\dagger} a_{l s}+ & \sum_{d=1,2} E_{d} a_{d}^{\dagger} a_{d}+\sum_{r, s^{\prime}} E_{r s^{\prime}} a_{r s^{\prime}}^{\dagger} a_{r s^{\prime}} \\
& +\left(\sum_{d, l, s} \Omega_{d l s} a_{l s}^{\dagger} a_{d}+\sum_{d, r, s^{\prime}} \Omega_{d r s^{\prime}} a_{r s^{\prime}}^{\dagger} a_{d}+H . c\right)+U_{C} a_{1}^{\dagger} a_{1} a_{2}^{\dagger} a_{2},
\end{aligned}
$$

where the spin indices, $s, s^{\prime}= \pm 1 / 2$ are related to different quantization axes (n and $\mathbf{n}^{\prime}$, Fig. 1), and $E_{l s}, E_{r s^{\prime}}$ denote the energy levels in the reservoirs. The Zeeman splitting of the dot is denoted by $E_{d}$, where $d=1,2$. The last two terms describe the tunneling transitions 
between the reservoirs and the dot states generated by both the tunneling couplings $\Omega$ and the Coulomb repulsion of two electrons inside the dot.

All parameters of the tunneling Hamiltonian (1) are related to the initial microscopic description of the system in the configuration space $(\boldsymbol{x})$. For instance, the coupling $\Omega_{d l s}$ is given by the Bardeen formula [17]

$$
\Omega_{d l s}=-\frac{1}{2 m} \int_{\boldsymbol{x} \in \Sigma_{l}} \phi_{d}(\boldsymbol{x}) \stackrel{\leftrightarrow}{\nabla} \chi_{l s}(\boldsymbol{x}) d \sigma,
$$

where $\phi_{d}(\boldsymbol{x})$ and $\chi_{l s}(\boldsymbol{x})$ are the electron wave functions inside the dot and the reservoir, respectively and $\Sigma_{l}$ is a surface inside the potential barrier that separates the dot from the left reservoir. Since the spin quantization axes in the dot and in the leads differ from each other, the transition matrix elements ( $\Omega$ 's) in Eq. (2) depend on the relative angles between the dot and the lead polarization axes $\left(\theta_{L}\right.$ and $\theta_{R}$ for left and right leads respectively). The simplest form of the couplings that respects $\mathrm{SU}(2)$ symmetry is

$$
\Omega_{d l s}=\Omega_{l} \mathrm{~d}_{s, s_{d}}^{(1 / 2)}\left(\theta_{L}\right) \quad \text { and } \quad \Omega_{d r s^{\prime}}=\Omega_{r} \mathrm{~d}_{s_{d}, s^{\prime}}^{(1 / 2)}\left(\theta_{R}\right) \text {, }
$$

where $s_{d}= \pm 1 / 2$ denotes the electron spin inside the dot, Fig. $1, \mathrm{~d}^{(1 / 2)}(\theta)$ is spin rotation matrix,

$$
\mathrm{d}^{1 / 2}(\theta)=\left(\begin{array}{cc}
\cos \frac{\theta}{2} & \sin \frac{\theta}{2} \\
-\sin \frac{\theta}{2} & \cos \frac{\theta}{2}
\end{array}\right),
$$

and $\Omega_{l / r}$ is spin-independent part of the couplings. Neglecting the energy dependence of these couplings, $\Omega_{l, r}=\Omega_{L, R}$, one can relate them to the partial widths (tunneling rates) as $\Gamma_{L, R}=2 \pi \Omega_{L, R}^{2} \rho_{L, R}$, where $\rho_{L, R}$ is density of the states in the left (right) reservoir.

In the case of large bias, $\left|E_{1,2}-\mu_{L, R}\right| \ll \Gamma_{L, R}$, the many-body Coulomb repulsion effects in the magneto-transport can be accounted for in the most simple and precise way by using the modified Bloch-type equations for the reduced density matrix $[15,16]$. These equations can be derived from the many-body Schrödinger equation by integrating out the reservoir states in the limit of weak or strong Coulomb repulsion, $U_{C} \ll \mu_{L}-E_{1}$ or $U_{C} \gg \mu_{L}-E_{1}$, without any stochastic or other approximations. In addition these equations are very useful for evaluating of the shot-noise power spectrum. 
In order to apply our method we first redefine the vacuum state, $|\mathbf{0}\rangle$, by identifying it with the initial state of the entire system. For instance we can identify it with empty dot, with the emitter and collector filled up to the chemical potentials $\mu_{L, R}$, respectively. We also assume that the electrons in the emitter are polarized along the $\mathbf{n}$-direction, Fig. 1 . The many-body wave function, describing the entire system can be written in the most general way as

$$
\begin{aligned}
|\boldsymbol{\Psi}(t)\rangle=\left[b_{0}(t)+\right. & \sum_{d, l, s} b_{d l s}(t) a_{d}^{\dagger} a_{l s}+\sum_{l, s, r, s^{\prime}} b_{r s^{\prime} l s}(t) a_{r s^{\prime}}^{\dagger} a_{l s} \\
& \left.+\sum_{l, s, \bar{l}, \bar{s}} b_{12 l s \bar{s} \bar{s}}(t) a_{1}^{\dagger} a_{2}^{\dagger} a_{l s} a_{\bar{l} \bar{s}}+\sum_{d, l, s, \bar{l}, \bar{s}, r, s^{\prime}} b_{d r s^{\prime} l s \bar{s} \bar{s} r}(t) a_{d}^{\dagger} a_{r s^{\prime}}^{\dagger} a_{l s} a_{\bar{l} \bar{s}}+\cdots\right]|\mathbf{0}\rangle,
\end{aligned}
$$

where $d=\{1,2\}$ denotes a state with one electron in the dot and $l s\left(r s^{\prime}\right)$ denote the electron level in the emitter (collector). The amplitudes $b_{\alpha}(t)$ of finding the entire system in the state " $\alpha$ " are obtained from the Schrödinger equation, $i \partial_{t}|\Psi(t)\rangle=\mathcal{H}|\Psi(t)\rangle$ with the initial condition $b_{\alpha}(0)=\delta_{\alpha, 0}$

Let us introduce the (reduced) density matrix $\sigma_{j j^{\prime}}^{m, n}(t)$, where $n, m$ denote the number of electrons arriving the right reservoir with the spin components $s^{\prime}= \pm 1 / 2$, respectively, Figs. 1,2. The lower indices, $j, j^{\prime}$ denote the discrete states of the quantum dot. For instance, in the case of non-interacting (or weakly interacting) electrons $j, j^{\prime}=\{0,1,2,3\}$, Fig. 2. This density matrix, $\sigma_{j j^{\prime}}^{m, n}(t)$ can be easily constructed from the amplitudes $b(t)$, Eq. (5). For example,

$$
\begin{aligned}
& \sigma_{00}^{0,0}(t)=\left|b_{0}(t)\right|^{2}, \quad \sigma_{11}^{0,0}(t)=\sum_{l}\left|b_{1 l 1 / 2}(t)\right|^{2}, \quad \sigma_{22}^{0,0}(t)=\sum_{l}\left|b_{2 l 1 / 2}(t)\right|^{2}, \quad \sigma_{33}^{0,0}(t)=\sum_{l, \bar{l}}\left|b_{21 l l}(t)\right|^{2}, \\
& \sigma_{12}^{0,0}(t)=\sum_{l} b_{1 l 1 / 2}(t) \mathbf{b}_{2 l 1 / 2}^{*}(t), \quad \sigma_{00}^{1,0}(t)=\sum_{l, r}\left|b_{1 l 1 / 2 r 1 / 2}(t)\right|^{2}, \quad \ldots
\end{aligned}
$$

The diagonal density matrix elements, $\sigma_{j j}^{n, m}$, are the probabilities of finding the system in one of the states shown in Fig. 2 and the off-diagonal matrix elements ("coherencies") describe a linear superposition of these states. 


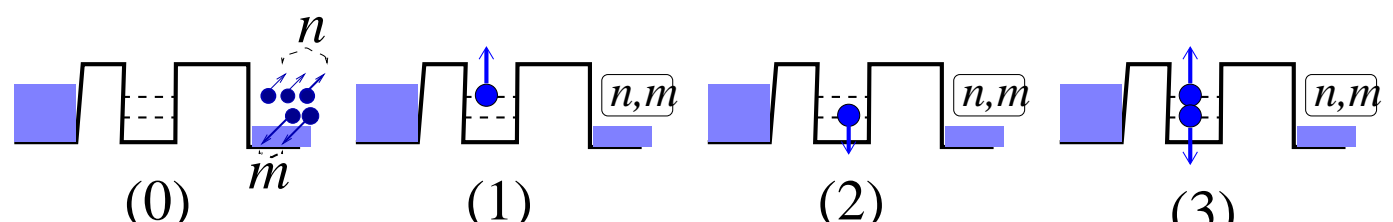

(0)
(1)
(2)

Fig. 2. Four available states of the quantum dot. The indices $n, m$ denote the number of electrons with the spin components $s^{\prime}= \pm 1 / 2$ in the right reservoir.

It was demonstrated in Ref. $[15,16]$ that the Schrödinger equation for the entire system, $i \partial_{t}|\Psi(t)\rangle=\mathcal{H}|\Psi(t)\rangle$, can be reduced to Bloch-type rate equations describing the reduced density-matrix $\sigma_{j j^{\prime}}^{n, m}(t)$. This reduction takes place after partial tracing over the reservoir states. It becomes exact in the limit of large bias without the explicit use of any Markov-type or weak coupling approximations [18]. In the general case these equations are [16]

$$
\begin{aligned}
& \dot{\sigma}_{j j^{\prime}}=i\left(E_{j^{\prime}}-E_{j}\right) \sigma_{j j^{\prime}}+i\left(\sum_{k} \sigma_{j k} \tilde{\Omega}_{k \rightarrow j^{\prime}}-\sum_{k} \tilde{\Omega}_{j \rightarrow k} \sigma_{k j^{\prime}}\right) \\
& -\sum_{k, k^{\prime}} \mathcal{P}_{2} \pi \rho\left(\sigma_{j k} \Omega_{k \rightarrow k^{\prime}} \Omega_{k^{\prime} \rightarrow j^{\prime}}+\sigma_{k j^{\prime}} \Omega_{k \rightarrow k^{\prime}} \Omega_{k^{\prime} \rightarrow j}\right)+\sum_{k, k^{\prime}} \mathcal{P}_{2} \pi \rho\left(\Omega_{k \rightarrow j} \Omega_{k^{\prime} \rightarrow j^{\prime}}+\Omega_{k \rightarrow j^{\prime}} \Omega_{k^{\prime} \rightarrow j}\right) \sigma_{k k^{\prime}},
\end{aligned}
$$

(for simplicity we have omitted the indices $m$ and $n$, which, however, can be easily restored from the conservation of the total number of electrons). Here $\Omega_{k \rightarrow k^{\prime}}$ denotes the singleelectron hopping amplitude that generates the $k \rightarrow k^{\prime}$ transition. We distinguish between the amplitudes $\tilde{\Omega}$ describing single-electron hopping among isolated states and $\Omega$ describing transitions among isolated and continuum states. The latter can generate transitions between the isolated states of the system, but only indirectly, via two consecutive jumps of an electron, into and out of the continuum reservoir states (with the density of states $\rho$ ). These transitions are represented by the third and the fourth terms of Eq. (7). The third term describes the transitions $\left(k \rightarrow k^{\prime} \rightarrow j\right)$ or $\left(k \rightarrow k^{\prime} \rightarrow j^{\prime}\right)$, which cannot change the number of electrons $(n, m)$ in the collector. The fourth term describes the transitions $(k \rightarrow j$ and $\left.k^{\prime} \rightarrow j^{\prime}\right)$ or $\left(k \rightarrow j^{\prime}\right.$ and $\left.k^{\prime} \rightarrow j\right)$ which increase the number of electrons in the collector by 
one. These two terms of Eq. (7) are analogues of the "loss" (negative) and the "gain" (positive) terms in the classical rate equations, respectively. Yet, the sign of these terms depends on the relative sign of the corresponding couplings $\Omega$ [1]. In our case it is determined by the sign of the spin-flip amplitude, Eq. (3). In addition, there is a (permutation) operator, $\mathcal{P}_{2}= \pm 1$, due to anti-commutation of the fermions operators, $a_{1,2}^{\dagger}$ in Eq. (5) (See also [15]). The prefactor $\mathcal{P}_{2}=-1$ whenever the loss or the gain terms in Eq. (7) are generated by a two-particle state of the dot. Otherwise $\mathcal{P}_{2}=1$.

\section{NON-INTERACTING ELECTRONS.}

Consider first the case of no electron repulsion inside the dot, $U_{C}=0$. (In fact, the results would be the same for $U_{C} \ll \mu_{L}-E_{1}$, assuming the couplings $\Omega$ are independent of energy). As in the previous section we choose the initial ("vacuum") state corresponding to the polarized electrons in the left reservoir, $s=1 / 2$ (Fig. 1). In this case all four configurations shown in Fig. 3 contribute to Eqs. (7). Taking into account that there is no

direct coupling between the states, $E_{1,2}$, i.e. $\tilde{\Omega}=0$, one obtains the following Bloch-type rate equations for the density matrix $\sigma_{j j^{\prime}}^{n, m}(t)$

$$
\begin{gathered}
\dot{\sigma}_{00}^{n, m}=-\Gamma_{L} \sigma_{00}^{n, m}+\Gamma_{R}^{(1)}\left(\sigma_{11}^{n-1, m}+\sigma_{22}^{n, m-1}\right)+\Gamma_{R}^{(2)}\left(\sigma_{11}^{n, m-1}+\sigma_{22}^{n-1, m}\right)+\Gamma_{L}^{(2)} \sigma_{11}^{n, m}+\Gamma_{L}^{(1)} \sigma_{22}^{n, m} \\
+\Gamma_{L}^{(12)}\left(\sigma_{12}^{n, m}+\sigma_{21}^{n, m}\right)-\Gamma_{R}^{(12)}\left(\sigma_{12}^{n-1, m}+\sigma_{21}^{n-1, m}-\sigma_{12}^{n, m-1}-\sigma_{21}^{n, m-1}\right) \\
\dot{\sigma}_{11}^{n, m}=-\left(\Gamma_{R}+2 \Gamma_{L}^{(2)}\right) \sigma_{11}^{n, m}+\Gamma_{L}^{(1)}\left(\sigma_{00}^{n, m}+\sigma_{33}^{n, m}\right)-\Gamma_{L}^{(12)}\left(\sigma_{12}^{n, m}+\sigma_{21}^{n, m}\right) \\
+\Gamma_{R}^{(2)} \sigma_{33}^{n-1, m}+\Gamma_{R}^{(1)} \sigma_{33}^{n, m-1} \\
\dot{\sigma}_{22}^{n, m}=-\left(\Gamma_{R}+2 \Gamma_{L}^{(1)}\right) \sigma_{22}^{n, m}+\Gamma_{L}^{(2)}\left(\sigma_{00}^{n, m}+\sigma_{33}^{n, m}\right)-\Gamma_{L}^{(12)}\left(\sigma_{12}^{n, m}+\sigma_{21}^{n, m}\right) \\
\quad+\Gamma_{R}^{(1)} \sigma_{33}^{n-1, m}+\Gamma_{R}^{(2)} \sigma_{33}^{n, m-1} \\
\dot{\sigma}_{33}^{n, m}=-\left(2 \Gamma_{R}+\Gamma_{L}\right) \sigma_{33}^{n, m}+\Gamma_{L}^{(2)} \sigma_{11}^{n, m}+\Gamma_{L}^{(1)} \sigma_{22}^{n, m}+\Gamma_{L}^{(12)}\left(\sigma_{12}^{n, m}+\sigma_{21}^{n, m}\right) \\
\dot{\sigma}_{12}^{n, m}=-(i \epsilon+\Gamma) \sigma_{12}^{n, m}-\Gamma_{L}^{(12)}\left(\sigma_{00}^{n, m}+\sigma_{11}^{n, m}+\sigma_{22}^{n, m}+\sigma_{33}^{n, m}\right)+\Gamma_{R}^{(12)}\left(\sigma_{33}^{n-1, m}-\sigma_{33}^{n, m-1}\right),
\end{gathered}
$$


where $\Gamma=\Gamma_{L}+\Gamma_{R}$ and $\Gamma_{L, R}^{(1)}=\Gamma_{L, R} \cos ^{2}\left(\theta_{L, R} / 2\right), \Gamma_{L, R}^{(2)}=\Gamma_{L, R} \sin ^{2}\left(\theta_{L, R} / 2\right), \Gamma_{L, R}^{(12)}=$ $\Gamma_{L, R} \sin \left(\theta_{L, R} / 2\right) \cos \left(\theta_{L, R} / 2\right)$ are the partial tunneling widths of the levels $E_{1,2}$.

We now trace the origin of each term in these equations taking as an example Eq. (8b), corresponding to $j=j^{\prime}=1$ in Eq.(7). The first term in this equation is a "loss" term generated by the transitions $1 \rightarrow 0 \rightarrow 1$ and $1 \rightarrow 3 \rightarrow 1$ in Eq. (7). corresponding to the following processes: (a) an electron at the level $E_{1}$ (Fig. 3(1)) tunnels to the right reservoir and back to the same state, with the rate $\Gamma_{R} ;(\mathrm{b})$ the same electron tunnels to the available continuum states of the left reservoir and back to the level $E_{1}$, with the rate $\Gamma_{L}^{(2)}=\Gamma_{L} \sin ^{2}\left(\theta_{L} / 2\right)$. This can proceed only via spin-flip, since the spin-up states in the left reservoir are occupied; (c) an electron from occupied states of the left reservoir tunnels to the unoccupied level $E_{2}$ of the dot and then back to the same state of the left reservoir, with the rate $\Gamma_{L}^{(2)}$.

The second term in Eq. (8b) is a "gain" term generated by the transitions $0 \rightarrow 1,0 \rightarrow 1$ and $3 \rightarrow 1,3 \rightarrow 1$ of an electron from the left reservoir to the level $E_{1}$ and from the level $E_{2}$ to the unoccupied (spin-down) continuum states of the left reservoir.

The third, "loss", term in Eq. (8b) is generated by the transitions $2 \rightarrow 0 \rightarrow 1$ and $2 \rightarrow 3 \rightarrow 1$ via the left reservoir. These transitions involve the following processes: (a) an electron at the level $E_{2}$ (Fig. $3(2)$ ) tunnels to an unoccupied, spin-down state of the left reservoir, and then makes a spin-flip transition to the state $E_{1}$ of the dot. The rate of this process is $(1 / 2) \Gamma_{L} \sin \left(\theta_{L}\right) \cos \left(\theta_{L} / 2\right)$, as follows from Eq. (7); (b) an electron from one of the occupied states of the left reservoir tunnels to the state $E_{1}$ with the corresponding amplitude $\Omega_{L} \cos \left(\theta_{L} / 2\right)$. Then an electron with energy $E_{2}$ tunnels to the vacant state of the left reservoir with the spin-flip amplitude $-\Omega_{L} \sin \left(\theta_{L} / 2\right)$. Since this transition proceeds via the two-electron state of the dot, the corresponding permutation prefactor, $\mathcal{P}_{2}=-1$. As a result, the rate of this (loss) process is $(1 / 2) \Gamma_{L} \sin \left(\theta_{L}\right)$. Similar transitions via the right reservoir cancel. Indeed the electron from energy level $E_{2}$ can reach the level $E_{1}$ by two ways: the first through the spin-flip hopping to the right reservoir and then to the level $E_{1}$ 
with no spin-flip, and the second, without spin-flip to the right reservoir, and then to the level $E_{1}$ with the spin flip. These two amplitudes are of the opposite sign.

The last two terms of Eq. (8b) are "gain" terms generated by the transitions $3 \rightarrow 1,3 \rightarrow 1$ of an electron from the state in Fig. 3(3) to the spin-up or spin-down states of the right reservoir. The number of electrons in the right reservoir increases by one.

\section{A. Resonant current in the collector}

Using Eqs. (8) we can easily obtain the spin-up and spin-down currents, $I_{1 / 2}(t)=$ $\sum_{n, m} n \dot{P}_{n, m}(t)$ and $I_{-1 / 2}(t)=\sum_{n, m} m \dot{P}_{n, m}(t)$, where $P_{n, m}(t)=\sum_{j=0}^{j=3} \sigma_{j j}^{n, m}$ is the probability of finding $n$ electrons with spin up and $m$ electrons with spin down in the right reservoir. One finds

$$
I_{ \pm 1 / 2}(t)=\Gamma_{R}\left[\frac{1 \pm \cos \theta_{R}}{2} \sigma_{11}(t)+\frac{1 \mp \cos \theta_{R}}{2} \sigma_{22}(t)+\sigma_{33}(t) \mp \frac{\sin \theta_{R}}{2}\left(\sigma_{12}(t)+\sigma_{21}(t)\right)\right]
$$

where $\sigma_{j j^{\prime}}(t)=\sum_{n, m} \sigma_{j j^{\prime}}^{n, m}(t)$. The latter can be obtained from the following matrix equation

$$
\dot{X}(t)+B X(t)=0
$$

obtained by the summation of Eqs. (8) over $n, m$. Here $X=\left\{\sigma_{00}, \sigma_{11}, \sigma_{22}, \sigma_{33}, \sigma_{12}, \sigma_{21}\right\}$ and $B$ is the corresponding $6 \times 6$ matrix,

$$
B=\left(\begin{array}{cccccc}
\Gamma_{L} & -\Gamma_{L}^{(2)}-\Gamma_{R} & -\Gamma_{L}^{(2)}-\Gamma_{R} & 0 & -\Gamma_{L}^{(12)} & -\Gamma_{L}^{(12)} \\
-\Gamma_{L}^{(1)} & \Gamma_{R}+2 \Gamma_{L}^{(2)} & 0 & -\Gamma_{L}^{(1)}-\Gamma_{R} & \Gamma_{L}^{(12)} & \Gamma_{L}^{(12)} \\
-\Gamma_{L}^{(2)} & 0 & \Gamma_{R}+2 \Gamma_{L}^{(1)} & -\Gamma_{L}^{(2)}-\Gamma_{R} & \Gamma_{L}^{(12)} & \Gamma_{L}^{(12)} \\
0 & -\Gamma_{L}^{(2)} & -\Gamma_{L}^{(1)} & \Gamma_{L}+2 \Gamma_{R} & -\Gamma_{L}^{(12)} & -\Gamma_{L}^{(12)} \\
\Gamma_{L}^{(12)} & \Gamma_{L}^{(12)} & \Gamma_{L}^{(12)} & \Gamma_{L}^{(12)} & i \epsilon+\Gamma & 0 \\
\Gamma_{L}^{(12)} & \Gamma_{L}^{(12)} & \Gamma_{L}^{(12)} & \Gamma_{L}^{(12)} & 0 & -i \epsilon+\Gamma
\end{array}\right)
$$

Solving Eqs. (10) and substituting the result into Eqs. (9) we find the following simple expressions for the average polarized current:

$$
\begin{aligned}
& I_{ \pm 1 / 2}(t)=\frac{\Gamma_{L} \Gamma_{R}}{2 \Gamma}\left(1 \pm \cos \theta_{L} \cos \theta_{R}\right)\left(1-e^{-\Gamma t}\right) \\
& \pm \frac{\Gamma_{L} \Gamma_{R} \Gamma \sin \theta_{L} \sin \theta_{R}}{2\left(\epsilon^{2}+\Gamma^{2}\right)}\left[1-e^{-\Gamma t} \cos (\epsilon t)+e^{-\Gamma t} \frac{\epsilon}{\Gamma} \sin (\epsilon t)\right] .
\end{aligned}
$$


The same result can be obtained in the framework of a single electron approach, valid for the noninteracting case. (See Appendix A.) As expected, the polarized resonant current displays damped oscillations. An example of these oscillations in $I_{1 / 2}(t)$ is shown in Fig. 3.

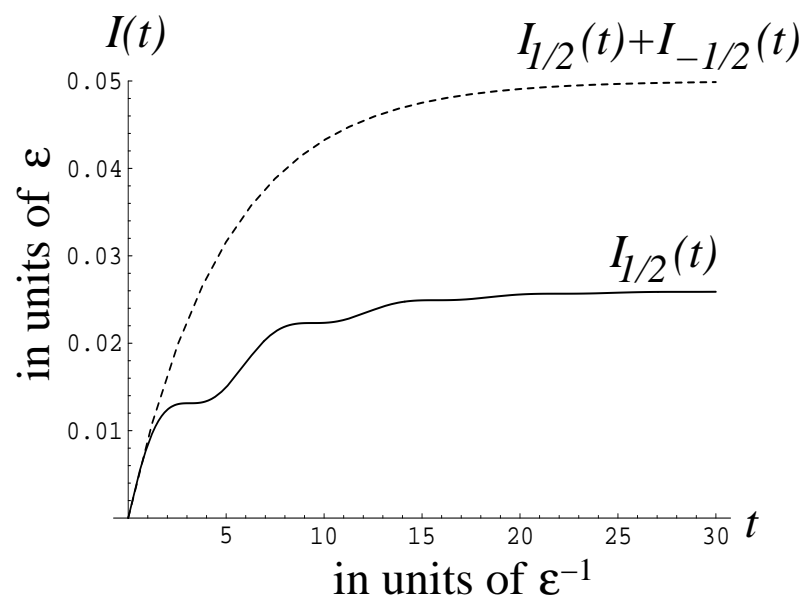

Fig. 3. Spin-up and total resonant currents through the Zeeman doublet as a function of time for $\theta_{L}=\theta_{R}=\pi / 2$ and $\Gamma_{L}=\Gamma_{R}=0.1 \epsilon$.

These oscillations, however, disappear in the total collector current,

$$
I(t)=I_{1 / 2}(t)+I_{-1 / 2}(t)=\frac{\Gamma_{L} \Gamma_{R}}{\Gamma}\left(1-e^{-\Gamma t}\right),
$$

even though electrons in the emitter are polarized.

\section{B. Noise spectrum of the total collector current}

Now we evaluate the noise-spectrum of the total current, represented by a sum of the spin-up and spin-down currents in the final state, Eq. (13). We introduce the density matrix $\sigma_{j j^{\prime}}^{N}(t)=\sum_{n} \sigma^{n, N-n}(t)$, obtained from Eqs. (8), where $N$ denotes the total number of electrons which have arrived at the right reservoir by time $t$. In order to calculate the shot-noise spectrum we use the McDonald formula [19]

$$
\left.S(\omega)=2 e^{2} \omega \int_{0}^{\infty} d t \sin (\omega t) \frac{d}{d t} \sum_{N} N^{2} P_{N}(t)\right]
$$


where $P_{N}(t)=\sum_{j=0}^{j=3} \sigma_{j j}^{N}(t)$. One easily finds from Eqs. (8) that

$$
\sum_{N} N^{2} \dot{P}_{N}(t)=\Gamma_{R} \sum_{N}(2 N+1)\left[\sigma_{11}^{N}(t)+\sigma_{22}^{N}(t)+2 \sigma_{33}^{N}(t)\right] .
$$

Substituting Eq. (15) into the McDonald formula, Eq. (14), we finally obtain

$$
S(\omega)=2 e^{2} \omega \Gamma_{R} \operatorname{Im}\left[Z_{11}(\omega)+Z_{22}(\omega)+2 Z_{33}(\omega)\right]
$$

where $Z(\omega)$ is a 6 -vector, $Z=\left\{Z_{00}, Z_{11}, Z_{22}, Z_{33}, Z_{12} Z_{21}\right\}$, defined as

$$
Z_{i j}(\omega)=\int_{0}^{\infty} \sum_{N}(2 N+1) \sigma_{i j}^{N}(t) \exp (i \omega t) d t
$$

One can find $Z_{i j}(\omega)$ directly from Eqs. (8) by performing the corresponding summation over $N$. As a result one obtains

$$
(B-i \omega I) Z(\omega)=\bar{X}+2 \Gamma_{R} \bar{Y}(\omega) .
$$

Here $B$ is given by Eq. (11) and $I$ is the unit matrix. The 6 -vector $\bar{X}$ corresponds to the stationary solution of Eqs. $(10), \bar{X}=X(t \rightarrow \infty)$ and $\bar{Y}(\omega)=\left\{Y_{11}+Y_{22}, Y_{33}, Y_{33}, 0,0,0\right\}$ where $Y(\omega)=\left\{Y_{00}, Y_{11}, Y_{22}, Y_{33}, Y_{33}, Y_{12}, Y_{21}\right\}$ is given by the equation

$$
(B-i \omega I) Y(\omega)=\bar{X}
$$

Using Eq. (18) we calculate the ratio of the shot-noise power spectrum to the Schottky noise, $S(\omega) / 2 e I$ (Fano factor), where $I=I(t \rightarrow \infty)=\Gamma_{L} \Gamma_{R} / \Gamma_{t}$, Eq.(13). In particular, the result has a simple analytical form for a symmetric dot, $\Gamma_{L}=\Gamma_{R}=\Gamma$. We find

$$
\frac{S(\omega)}{2 e I}=\frac{2 \Gamma^{2}+\omega^{2}}{4 \Gamma^{2}+\omega^{2}}+\frac{\Gamma^{2} \epsilon^{2} \sin ^{2} \theta_{L}}{\left(4 \Gamma^{2}+\omega^{2}\right)\left(4 \Gamma^{2}+\omega^{2}\right)} .
$$

As expected the shot-noise spectrum does not display any peak or dip at frequencies corresponding to the Zeeman splitting, since the interference effects are canceled in the total collector current. Yet the noise spectrum depends on the initial polarization of incoming electrons $\left(\theta_{L}\right)$, whereas the total collector current does not (see Eq. (13)). If electrons are initially polarized along the magnetic field inside the dot $(\overline{\mathbf{n}})$, the Fano factor is the same as in the case of resonant tunneling through a single level [20]. With increasing $\theta_{L}$, however, the current flows through both levels of the Zeeman doublet. This leads to an additional contribution to the shot noise, described by the second term of Eq. (20). 


\section{Ferromagnetic reservoirs}

Let us consider ferromagnetic reservoirs polarized along $\mathbf{n}$ and $\mathbf{n}$ ' directions, Fig. 1. In this case the rate equations (8) have to be modified since there are no available spin-down states in the left and right reservoirs. One easily obtains the following rate equations for the density matrix $\sigma_{j j^{\prime}}^{n}(t)$, where $n$ denotes the number of electron, arriving at the collector before time $t$ :

$$
\begin{aligned}
& \dot{\sigma}_{00}^{n}=-\Gamma_{L} \sigma_{00}^{n}+\Gamma_{R}^{(1)} \sigma_{11}^{n-1}+\Gamma_{R}^{(2)} \sigma_{22}^{n-1}-\Gamma_{R}^{(12)}\left(\sigma_{12}^{n-1}+\sigma_{21}^{n-1}\right) \\
& \dot{\sigma}_{11}^{n}=-\left(\Gamma_{L}^{(2)}+\Gamma_{R}^{(1)}\right) \sigma_{11}^{n}+\Gamma_{L}^{(1)} \sigma_{00}^{n}-\frac{1}{2}\left(\Gamma_{L}^{(12)}-\Gamma_{R}^{(12)}\right)\left(\sigma_{12}^{n}+\sigma_{21}^{n}\right)+\Gamma_{R}^{(2)} \sigma_{33}^{n-1} \\
& \dot{\sigma}_{22}^{n}=-\left(\Gamma_{L}^{(1)}+\Gamma_{R}^{(2)}\right) \sigma_{22}^{n}+\Gamma_{L}^{(2)} \sigma_{00}^{n}-\frac{1}{2}\left(\Gamma_{L}^{(12)}-\Gamma_{R}^{(12)}\right)\left(\sigma_{12}^{n}+\sigma_{21}^{n}\right)+\Gamma_{R}^{(1)} \sigma_{33}^{n-1} \\
& \dot{\sigma}_{33}^{n}=-\Gamma_{R} \sigma_{33}^{n}+\Gamma_{L}^{(2)} \sigma_{11}^{n}+\Gamma_{L}^{(1)} \sigma_{22}^{n}+\Gamma_{L}^{(12)}\left(\sigma_{12}^{n}+\sigma_{21}^{n}\right) \\
& \dot{\sigma}_{12}^{n}=-\left(i \epsilon+\frac{1}{2} \Gamma\right) \sigma_{12}^{n}-\Gamma_{L}^{(12)} \sigma_{00}^{n}-\frac{1}{2}\left(\Gamma_{L}^{(12)}-\Gamma_{R}^{(12)}\right)\left(\sigma_{11}^{n}+\sigma_{22}^{n}\right)+\Gamma_{R}^{(12)} \sigma_{33}^{n-1}
\end{aligned}
$$

Using these equations we first evaluate the average current, $I(t) \equiv I_{1 / 2}(t)$ given by Eq. (9) with $\sigma_{j j^{\prime}}(t)=\sum_{n} \sigma_{j j^{\prime}}^{n}(t)$. The latter quantities are obtained from a summation of Eqs. (21) over $n$. As a result Eqs. (21) are reduced to the matrix equation (10), where $B$ is the corresponding $6 \times 6$ matrix of the coefficients of Eqs. (21). Solving this equation we find the average current $I(t)$. For instance, in the case of $\theta_{L}=\theta_{R}$ one finds for the stationary current, $I=I(\infty)=\Gamma_{L} \Gamma_{R} /\left(\Gamma_{L}+\Gamma_{R}\right)$. One obtains the same expression for the resonant tunneling of unpolarized electrons through a single level.

The time dependence of the average current, $I(t)$, is displayed in Fig. 4 for symmetric and asymmetric dots, $\Gamma_{L}=\Gamma_{R}=0.1 \epsilon$ and $\Gamma_{L}=\epsilon, \Gamma_{R}=0.1 \epsilon$, respectively. Comparing with Fig. 2 one finds that the oscillations in the average current are more pronounced in the case of ferromagnetic reservoirs. This can be anticipated since the corresponding spin-flip transitions via the spin-down states of the reservoirs do not exist. We recall that precisely these transitions resulted in the cancelation of the interference effects in the previous case. 


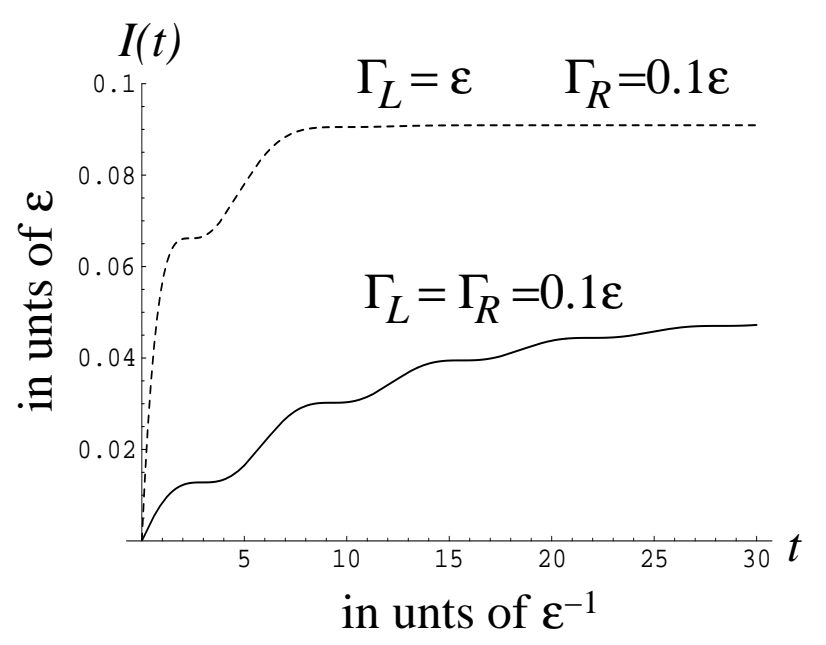

Fig. 4. The polarized resonant current through the Zeeman doublet with ferromagnetic reservoirs and $\theta_{L}=\theta_{R}=\pi / 2$. The solid line corresponds to $\Gamma_{L}=\Gamma_{R}=0.1 \epsilon$ and the dashed line to $\Gamma_{L}=\epsilon$ and $\Gamma_{R}=0.1 \epsilon$

Now we can evaluate the shot-noise spectrum, $S(\omega)$ using the McDonald formula. One obtains from Eqs. (14) and (21)

$$
S(\omega)=2 e^{2} \omega \operatorname{Im}\left\{\Gamma_{R}^{(1)} Z_{11}(\omega)+\Gamma_{R}^{(2)} Z_{22}(\omega)+\Gamma_{R} Z_{33}(\omega)-\Gamma_{R}^{(12)}\left[Z_{12}(\omega)+Z_{21}(\omega)\right]\right\}
$$

where $Z(\omega)$ is given by Eq. (18) with the matrix $B$ corresponding to Eqs. (21) and $\bar{Y}=\left\{\bar{Y}_{00}, \bar{Y}_{11}, \bar{Y}_{22}, 0, \bar{Y}_{12}, \bar{Y}_{21}\right\}$. Here $\bar{Y}_{00}=\cos ^{2} \frac{\theta_{R}}{2} Y_{11}+\sin ^{2} \frac{\theta_{R}}{2} Y_{22}-\frac{\sin \theta_{R}}{2}\left(Y_{12}+Y_{21}\right)$, $\bar{Y}_{11}=\sin ^{2} \frac{\theta_{R}}{2} Y_{33}, \bar{Y}_{22}=\cos ^{2} \frac{\theta_{R}}{2} Y_{33}$, and $\bar{Y}_{12}=\bar{Y}_{21}=\frac{\sin \theta_{R}}{2} Y_{33}$, while $Y_{j j^{\prime}}=Y_{j j^{\prime}}(\omega)$ are given by Eq. (19).

The corresponding Fano factor is shown in Fig. 5 for the same parameters as in Fig. 4. It clearly displays a dip at the Zeeman frequency for a symmetric dot. It reflects the damped oscillations in the average current, shown in Fig. 4. The dip, however, almost disappears for an asymmetric dot with large $\Gamma_{L}$. 


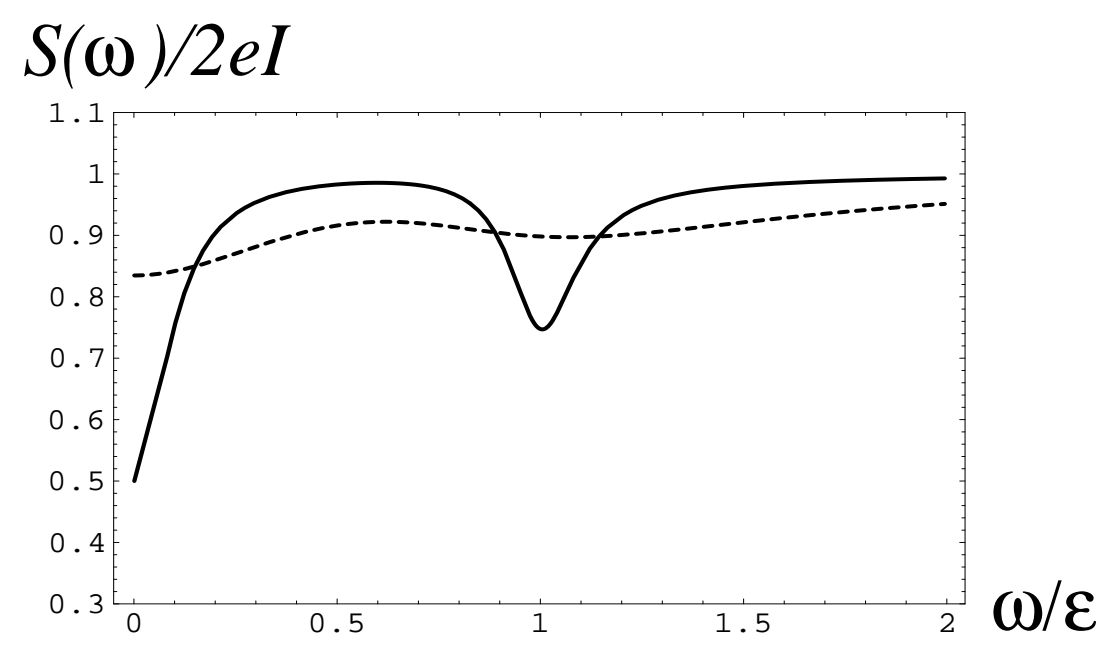

Fig. 5. The Fano factor versus $\omega$ for a polarized electron current with ferromagnetic reservoirs and $\theta_{L}, \theta_{R}=\pi / 2$. The solid line corresponds to $\Gamma_{L}=\Gamma_{R}=0.1 \epsilon$ and the dashed line to $\Gamma_{L}=\epsilon$ and $\Gamma_{R}=0.1 \epsilon$

\section{COULOMB BLOCKADE}

We now introduce strong Coulomb repulsion inside the dot, $U_{C} \gg \mu_{L}-E_{1}$, so that the state (3) in Fig. 3 is not available. As a result the corresponding rate equations have an even simpler form than those found for non-interacting electrons. Consider again the case of ferromagnetic reservoirs, where the quantum interference effects are most pronounced. The corresponding rate equations for the case of Coulomb blockade can be obtained from Eqs. (21) for non-interacting electrons, by eliminating configurations with two electrons in the dot. In the following we consider separately the electron current in the right and in the left reservoirs.

\section{A. Collector current}

The electrical current in the right reservoir and its power spectrum are obtained from the following rate equation 


$$
\begin{aligned}
& \dot{\sigma}_{00}^{n}=-\Gamma_{L} \sigma_{00}^{n}+\Gamma_{R}^{(1)} \sigma_{11}^{n-1}+\Gamma_{R}^{(2)} \sigma_{22}^{n-1}-\Gamma_{R}^{(12)}\left(\sigma_{12}^{n-1}+\sigma_{21}^{n-1}\right) \\
& \dot{\sigma}_{11}^{n}=-\Gamma_{R}^{(1)} \sigma_{11}^{n}+\Gamma_{L}^{(1)} \sigma_{00}^{n}+\frac{\Gamma_{R}^{(12)}}{2}\left(\sigma_{12}^{n}+\sigma_{21}^{n}\right) \\
& \dot{\sigma}_{22}^{n}=-\Gamma_{R}^{(2)} \sigma_{22}^{n}+\Gamma_{L}^{(2)} \sigma_{00}^{n}+\frac{\Gamma_{R}^{(12)}}{2}\left(\sigma_{12}^{n}+\sigma_{21}^{n}\right) \\
& \dot{\sigma}_{12}^{n}=-\left(i \epsilon+\frac{\Gamma_{R}}{2}\right) \sigma_{12}^{n}-\Gamma_{L}^{(12)} \sigma_{00}^{n}+\frac{\Gamma_{R}^{(12)}}{2}\left(\sigma_{11}^{n}+\sigma_{22}^{n}\right)
\end{aligned}
$$

Using these equations one finds for the average (polarized) current in the collector

$$
I_{R}(t)=\Gamma_{R}^{(1)} \sigma_{11}(t)+\Gamma_{R}^{(2)} \sigma_{22}(t)-\Gamma_{R}^{(12)}\left[\sigma_{12}(t)+\sigma_{21}(t)\right]
$$

where the $\sigma_{j j^{\prime}}(t)=\sum_{n, m} \sigma_{j j^{\prime}}^{n, m}(t)$ are obtained from Eq. (10) for $X=\left\{\sigma_{00}, \sigma_{11}, \sigma_{22}, \sigma_{12}, \sigma_{21}\right\}$, and $B$ is the $5 \times 5$ matrix obtained from the coefficients of Eqs. (23). Solving such a modified Eq. (10) for $\theta_{L}=\theta_{R}$, one finds for the stationary current,

$$
I_{R}=I_{R}(\infty)=\frac{\Gamma_{L} \Gamma_{R}}{2 \Gamma_{L}+\Gamma_{R}}
$$

This expression shows an asymmetry with respect to the widths $\Gamma_{L}$ and $\Gamma_{R}$, in contrast with the non-interacting case. The reason is that an electron enters the dot from the left reservoir with the rate $2 \Gamma_{L}$. However, it leaves it with the rate $\Gamma_{R}$, since the state with two levels of the dot occupied is forbidden.

The shot-noise power spectrum for the collector current is given by

$$
S_{R}(\omega)=2 e^{2} \omega \operatorname{Im}\left\{\Gamma_{R}^{(1)} Z_{11}(\omega)+\Gamma_{R}^{(2)} Z_{22}(\omega)-\Gamma_{R}^{(12)}\left[Z_{12}(\omega)+Z_{21}(\omega)\right]\right\}
$$

Here $Z_{i j}(\omega)$ are obtained from Eqs. (18),(19), where $\bar{Y}=\left\{\bar{Y}_{00}, 0,0,0,0\right\}$ and $\bar{Y}_{00}=$ $\cos ^{2} \frac{\theta_{R}}{2} Y_{11}+\sin ^{2} \frac{\theta_{R}}{2} Y_{22}-\frac{\sin \theta_{R}}{2}\left(Y_{12}+Y_{21}\right)$.

The results of our calculations of $S(\omega)$ for symmetric and asymmetric quantum dots in the case of Coulomb blockade are shown in Fig. 6. 


\section{$S(\omega) / 2 e I$}

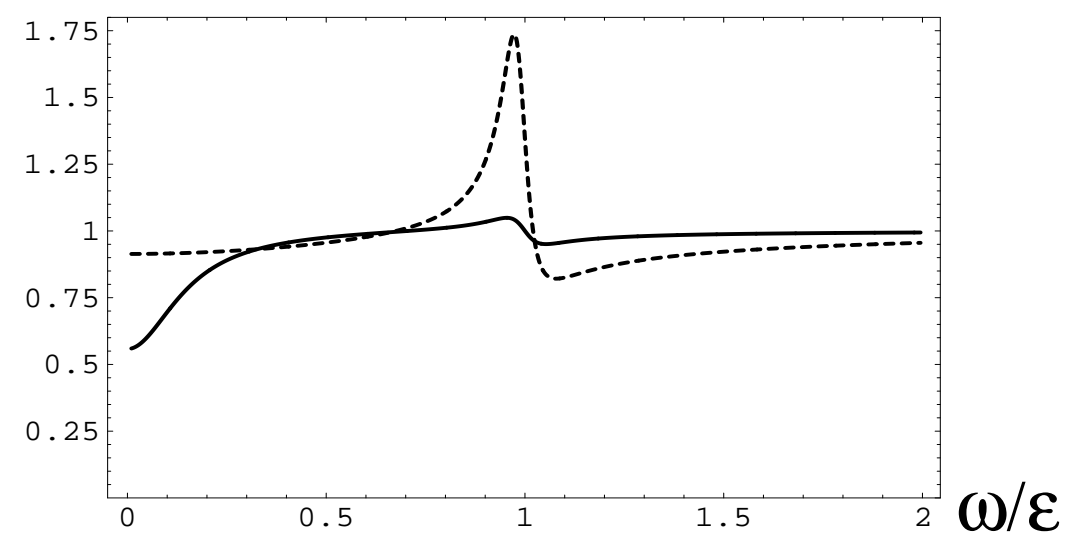

Fig. 6. The Fano factor versus $\omega$ for a polarized collector current with ferromagnetic reservoirs and Coulomb blockade and $\theta_{L}, \theta_{R}=\pi / 2$. The solid line corresponds to $\Gamma_{L}=\Gamma_{R}=0.1 \epsilon$ and the dashed line to $\Gamma_{L}=\epsilon$ and $\Gamma_{R}=0.1 \epsilon$.

\section{B. Emitter current}

We now consider the electric current and its power spectrum in the left reservoir. These

quantities are determined from the density-matrix $\sigma_{j j^{\prime}}^{p}$, where $p$ is the number of electrons that left the emitter before time $t$, (the number of holes in the left reservoir). The corresponding rate equations are similar to Eqs. (23). One finds

$$
\begin{aligned}
& \dot{\sigma}_{00}^{p}=-\Gamma_{L} \sigma_{00}^{p}+\Gamma_{R}^{(1)} \sigma_{11}^{p}+\Gamma_{R}^{(2)} \sigma_{22}^{p}-\Gamma_{R}^{(12)}\left(\sigma_{12}^{p}+\sigma_{21}^{p}\right) \\
& \dot{\sigma}_{11}^{p}=-\Gamma_{R}^{(1)} \sigma_{11}^{p}+\Gamma_{L}^{(1)} \sigma_{00}^{p-1}+\frac{\Gamma_{R}^{(12)}}{2}\left(\sigma_{12}^{p}+\sigma_{21}^{p}\right) \\
& \dot{\sigma}_{22}^{p}=-\Gamma_{R}^{(2)} \sigma_{22}^{p}+\Gamma_{L}^{(2)} \sigma_{00}^{p-1}+\frac{\Gamma_{R}^{(12)}}{2}\left(\sigma_{12}^{p}+\sigma_{21}^{p}\right) \\
& \dot{\sigma}_{12}^{p}=-\left(i \epsilon+\frac{\Gamma_{R}}{2}\right) \sigma_{12}^{p}-\Gamma_{L}^{(12)} \sigma_{00}^{p-1}+\frac{\Gamma_{R}^{(12)}}{2}\left(\sigma_{11}^{p}+\sigma_{22}^{p}\right)
\end{aligned}
$$

The average emitter current in the left reservoir is given by $I_{L}(t)=\Gamma_{L} \sigma_{00}(t)$, which differs from Eq. (24) describing the collector current, $I_{R}(t)$. Yet, as expected, their stationary values 
coincide, $I_{L}(\infty)=I_{R}(\infty)$, Eq. (25).

The shot-noise power spectrum of the emitter current is given

$$
S_{L}(\omega)=2 e^{2} \omega \Gamma_{L} \operatorname{Im} Z_{00}(\omega)
$$

instead of Eq. (26) for $S_{R}(\omega)$, where $Z_{i j}(\omega)$ are obtained from Eqs. (18),(19). Yet, $\bar{Y}(\omega)=$ $\left\{0, \cos ^{2} \frac{\theta_{L}}{2}, \sin ^{2} \frac{\theta_{L}}{2},-\frac{\sin \theta_{L}}{2},-\frac{\sin \theta_{L}}{2}\right\} Y_{00}(\omega)$, in contrast with the corresponding expression for $S_{R}(\omega)$. Even though the expressions for $S_{L, R}(\omega)$ are quite different, one finds that the shotnoise power of the emitter current is the same as that in the collector current, $S_{L}(\omega)=S_{R}(\omega)$.

\section{Circuit current}

In general the circuit current is given by $I_{c}(t)=\alpha I_{L}(t)+\beta I_{R}(t)$, where the coefficients $\alpha, \beta$ with $\alpha+\beta=1$ depend on the junction capacities [21]. Using charge conservation, $I_{L}=I_{R}+\dot{Q}$, where $Q$ is charge in the dot, one finds

$$
I_{c}(t) I_{c}(0)=\alpha I_{L}(t) I_{L}(0)+\beta I_{R}(t) I_{R}(0)-\alpha \beta \dot{Q}(t) \dot{Q}(0) .
$$

Using this relation one finds a simple expression for the noise spectrum of the circuit current $[4,22]$

$$
S_{c}(\omega)=\alpha S_{L}(\omega)+\beta S_{R}(\omega)-\alpha \beta \omega^{2} S_{Q}(\omega)
$$

where $S_{Q}(\omega)$ is Fourier transform of the charge correlation function. This quantity can be obtained straightforwardly from the matrix equation (19), where $\bar{X}$ is the 5-vector $\left\{0, \sigma_{11}(\infty), \sigma_{22}(\infty), 0,0\right\}$. Then $S_{Q}(\omega)=4 \operatorname{Re}\left[Y_{11}(\omega)+Y_{22}(\omega)\right]$

The results of our calculations of $S_{c}(\omega)$ for $\alpha=\beta=1 / 2$ are shown in Fig. 7 . 


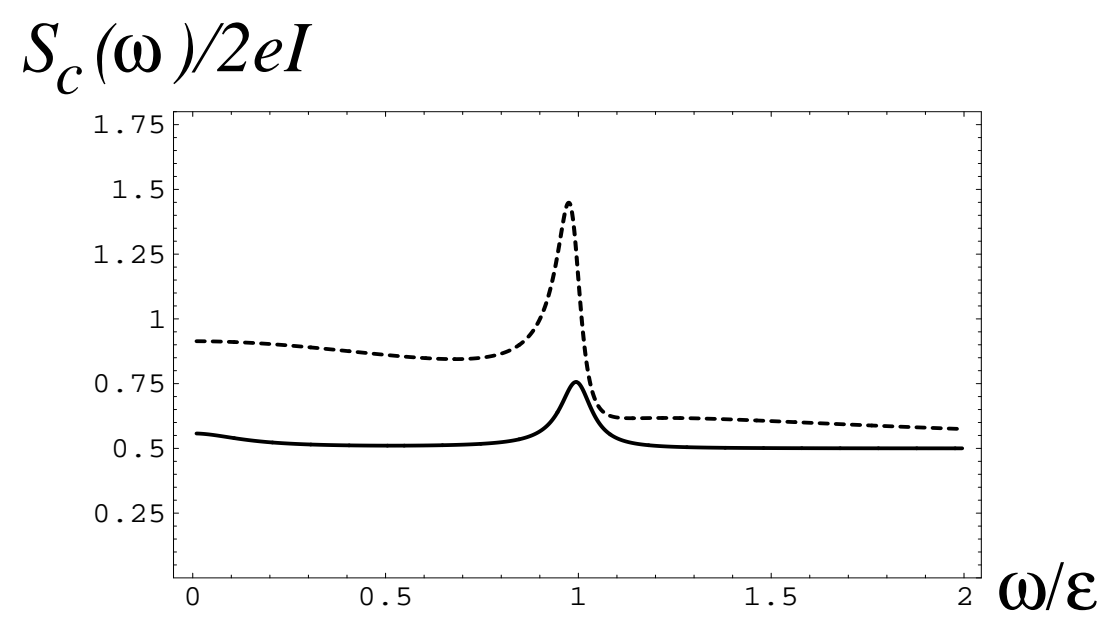

Fig. 7. The Fano factor for the circuit $(\alpha=\beta=1 / 2)$ versus $\omega$ for a polarized electron current with ferromagnetic reservoirs and Coulomb blockade and $\theta_{L}, \theta_{R}=\pi / 2$. The solid line corresponds to $\Gamma_{L}=\Gamma_{R}=0.1 \epsilon$ and the dashed line to $\Gamma_{L}=\epsilon$ and $\Gamma_{R}=0.1 \epsilon$.

One finds from Figs. 6 and 7 that the Coulomb blockade modifies the current spectrum very drastically with respect to the non-interacting case, Fig. 5.

\section{CONCLUSIONS}

In this paper, we study the interference effects in magneto-transport through Zeeman split levels of quantum dots or impurities. We concentrated on the time-dependent properties and the power spectrum of the electric current by applying the new approach using quantum rate equations, which is mostly suitable for this type of problems. We explicitly demonstrated that our method produces the same results as a single electron approach, widely used for a description of non-interacting electron transport. Yet the quantum rate equations method is valid also for the case of interacting electrons and accounts for the Coulomb blockade in the most simple and precise way.

Our results indicate that the Coulomb blockade plays an important role in the spectral properties of the transport current. First of all, in the presence of Coulomb blockade the 
signal-to-noise ratio significantly is amplified, as one can observe from the results in the previous sections. This is probably a consequence of the prohibition of double occupation of the resonant level in the quantum dot. Indeed, when two electrons in the dot are present, the interference effects are suppressed due to the "randomization" of the relative phase. Interestingly, the dip in the noise spectrum for the noninteracting electrons is replaced by a peak as result of Coulomb interaction. Clearly the Coulomb interaction modifies the phase of the electrons tunneling trough the dot, which "flips" the spectral feature in the noise. The details of this very interesting phenomena must be studied in the future.

We emphasize that the coherent oscillations in the current can be observed only for polarized current and that oscillations disappear for unpolarized current. This is different from the resonant transport through two orbital levels of a quantum dot or impurity, where the quantum interference effects can be observed even in unpolarized case. Therefore it is most natural to use ferromagnetic leads for observation and utilization of quantum interference effect in the magneto-transport. Thus our calculations were mostly concentrated on this

case. Our results show explicitly the appearance of peak or dip at a frequency near the Zeemann splitting frequency (Larmour frequency). We believe that this phenomenon can be useful for analyzing the noise spectroscopy of quantum dots or impurities. Indeed, the Zeeman splitting of a localized quantum dot orbital must be sensitive to local magnetic fields, and therefore one can hope that such coherent effect, if observed experimentally, may allow for detection of the local hyperfine structure of the dot/impurity. This, however, must be a subject of a separate investigation.

\section{ACKNOWLEDGEMENT}

We thank J. Brown, L. Fedichkin, M. B. Hastings, M. Hawley, and I. Martin for valuable discussions. We are especially grateful to Gary D. Doolen for important remarks and for proofreading the manuscript. The work was supported by the Department of Energy under Contract No. W-7405-ENG-36 and by DOE Office of basic Energy Sciences. D. M. was 
supported, in part, by the US NSF grant DMR-0121146.

\section{APPENDIX A: SINGLE-ELECTRON DESCRIPTION}

In the case of non-interacting electrons one can compare our results with those obtained using a single-electron approach. Although the latter is widely used in the literature, it is usually restricted to the time-independent (stationary) case. Here we present an extension of the single-electron approach for the non-stationary case. This would allow us to evaluate the time-dependent resonant current, Fig. 3, and to compare the results with those obtained from Eqs. (7).

Let us consider a system consisting of the reservoirs and the quantum dot filled with only a single electron. We assume that this electron is initially in the left reservoir (emitter) at the level $E_{\bar{l}}$ with the spin polarized along the $\mathbf{n}$-direction, Fig. 1 . The electron motion is described by a wave function which can be written in the most general way as [23]:

$$
|\Psi(t)\rangle=\left[\sum_{l, s} \mathrm{~b}_{l s}(t) a_{l s}^{\dagger}+\sum_{d=1,2} \mathrm{~b}_{d}(t) a_{d}^{\dagger}+\sum_{r, s^{\prime}} \mathrm{b}_{r s^{\prime}}(t) a_{r s^{\prime}}^{\dagger}\right]|0\rangle,
$$

where $b_{\alpha}(t)$ is the amplitude of finding the electron in the state $\alpha$ given by a corresponding creation operator. These amplitudes are obtained from the Schrödinger equation $|\Psi(t)\rangle$, with the initial conditions $\mathrm{b}_{l s}(0)=\delta_{l, \bar{l}} \delta_{s, 1 / 2}$ and $\mathrm{b}_{d}(0)=\mathrm{b}_{r s}(0)=0$. It is useful to use the Laplace transform, $\tilde{\mathrm{b}}(E)=\int_{0}^{\infty} \mathrm{b}(t) \exp (i E t) d t$. In this case the time-dependent Schrödinger

equation for the amplitudes $\tilde{\mathrm{b}}(E)$ becomes the following system of linear algebraic equations

$$
\begin{aligned}
& \left(E-E_{l s}\right) \tilde{\mathrm{b}}_{l s}(E)-\Omega_{l} \sum_{d^{\prime}=1,2} \mathrm{~d}_{s, s_{d^{\prime}}}^{(1 / 2)}\left(\theta_{L}\right) \tilde{\mathrm{b}}_{d^{\prime}}(E)=i \delta_{l, \bar{l}} \delta_{s, 1 / 2} \\
& \left(E-E_{d}\right) \tilde{\mathrm{b}}_{d}(E)-\sum_{l, s} \Omega_{l} \mathrm{~d}_{s_{d}, s}^{(1 / 2)}\left(\theta_{L}\right) \tilde{\mathrm{b}}_{l s}(E)-\sum_{r, s^{\prime}} \Omega_{r} \mathrm{~d}_{s_{d}, s^{\prime}}^{(1 / 2)}\left(\theta_{R}\right) \tilde{\mathrm{b}}_{r s^{\prime}}(E)=0 \\
& \left(E-E_{r s^{\prime}}\right) \tilde{\mathrm{b}}_{r s^{\prime}}(E)-\Omega_{r} \sum_{d^{\prime}=1,2} \mathrm{~d}_{s^{\prime}, s_{d^{\prime}}}^{(1 / 2)}\left(\theta_{R}\right) \tilde{\mathrm{b}}_{d^{\prime}}(E)=0 .
\end{aligned}
$$

Substituting $\tilde{\mathrm{b}}_{l s}$ and $\tilde{\mathrm{b}}_{r s^{\prime}}$ from Eqs. (A2a), (A2c) into Eq. (A2b) and replacing the sums on $l$ and $r$ by the integrals, we obtain 


$$
\begin{aligned}
& \left(E-E_{1}+i \frac{\Gamma_{L}+\Gamma_{R}}{2}\right) \tilde{b}_{1}(E)=i \frac{\Omega_{L} \cos \left(\theta_{L} / 2\right)}{E-E_{\bar{l}, 1 / 2}} \\
& \left(E-E_{2}+i \frac{\Gamma_{L}+\Gamma_{R}}{2}\right) \tilde{b}_{2}(E)=-i \frac{\Omega_{L} \sin \left(\theta_{L} / 2\right)}{E-E_{\bar{l}, 1 / 2}} .
\end{aligned}
$$

Note that the amplitudes, $\tilde{\mathrm{b}}_{1}(E)$ and $\tilde{\mathrm{b}}_{2}(E)$, are decoupled in Eqs. (A3) although the corresponding states are connected via the continuum. The reason is that the spin-flip couplings of the dot with the reservoirs are of the opposite sign for the spin-up and the spin-down states of the $\operatorname{dot}\left(E_{1}\right.$ and $E_{2}$ in Fig. 1). However, for the general case of resonant tunneling through two levels, the corresponding amplitudes are coupled via the interaction through continuum [1].

Using the inverse Laplace transform $\mathbf{b}_{1,2}(t)=\int \tilde{\mathbf{b}}_{1,2}(E) \exp (-i E t) d E /(2 \pi)$, we obtain for the amplitudes, $b_{1,2}(t)$, for finding the electron inside the dot

$$
\begin{aligned}
& \mathrm{b}_{1}(t)=\frac{\Omega_{L} \cos \left(\theta_{L} / 2\right)}{E_{L}-E_{1}+i \frac{\Gamma}{2}}\left(e^{-i E_{L} t}-e^{-i E_{1} t-\frac{\Gamma}{2} t}\right) \\
& \mathrm{b}_{2}(t)=-\frac{\Omega_{L} \sin \left(\theta_{L} / 2\right)}{E_{L}-E_{2}+i \frac{\Gamma}{2}}\left(e^{-i E_{L} t}-e^{-i E_{2} t-\frac{\Gamma}{2} t}\right),
\end{aligned}
$$

where $\Gamma=\Gamma_{L}+\Gamma_{R}$. The probability amplitude of finding the electron inside the collector is $\tilde{\mathrm{b}}_{r s^{\prime}}(t)=\int \tilde{\mathrm{b}}_{r s^{\prime}}(E) \exp (-i E t) d E /(2 \pi)$, where $\tilde{\mathbf{b}}_{r, s^{\prime}}(E)$ is given by Eq. (A2c)

$$
\tilde{\mathrm{b}}_{r s^{\prime}}(E)=\frac{\Omega_{R}}{E-E_{r s^{\prime}}} \sum_{d} \mathrm{~d}_{s^{\prime} s_{d}}^{(1 / 2)}\left(\theta_{R}\right) \tilde{\mathrm{b}}_{d}(E) .
$$

The above equations determine the motion of a single electron placed initially in the emitter. In order to obtain the polarized current, $I_{s^{\prime}}(t)$, in the single-electron model one has to sum over all initially occupied states $E_{\bar{l}}$ of the emitter and over all available states $E_{r}$ of the collector. Thus $I_{s^{\prime}}=d N_{s^{\prime}}(t) / d t$, where $N_{s^{\prime}}(t)=\sum_{\bar{l}, r}\left|\mathrm{~b}_{r s^{\prime}}(t)\right|^{2}$ is the average number of electrons with spin-up and spin-down $\left(s^{\prime}= \pm 1 / 2\right)$, accumulated in the collector by the time $t$. Using the inverse Laplace transform and replacing $\sum_{\bar{l}, r} \rightarrow \int \rho_{L} \rho_{R} d E_{L} d E_{R}$ we obtain

$$
N_{s^{\prime}}(t)=\int \rho_{L} \rho_{R} d E_{L} d E_{R} \int \frac{d E d E^{\prime}}{(2 \pi)^{2}} \tilde{\mathbf{b}}_{r s^{\prime}}(E) \tilde{\mathrm{b}}_{r s^{\prime}}^{*}\left(E^{\prime}\right) e^{i\left(E^{\prime}-E\right) t}
$$

Substituting Eq. (A5) into Eq. (A6) and integrating over $E_{r s^{\prime}}$ one obtains for the polarized current 


$$
\begin{gathered}
I_{1 / 2}(t)=\Gamma_{R} \int_{\mu_{R}}^{\mu_{L}} \rho_{L} d E_{L}\left|\cos \left(\theta_{R} / 2\right) \mathrm{b}_{1}(t)-\sin \left(\theta_{R} / 2\right) \mathrm{b}_{2}(t)\right|^{2} \\
I_{-1 / 2}(t)=\Gamma_{R} \int_{\mu_{R}}^{\mu_{L}} \rho_{L} d E_{L}\left|\sin \left(\theta_{R} / 2\right) \mathrm{b}_{1}(t)+\cos \left(\theta_{R} / 2\right) \mathrm{b}_{2}(t)\right|^{2}
\end{gathered}
$$

where the amplitudes $b_{1,2}(t)$ are given by Eqs. (A4). Note that these amplitudes in the stationary limit, $\mathrm{b}_{1,2}(t \rightarrow \infty)$, are the transmission amplitudes describing the resonance tunneling through the levels $E_{1,2}[7]$. Thus Eqs. (A7) represent a generalization of the Landauer formula for the time-dependent case.

For large bias, $\mu_{L}-\mu_{R} \gg \Gamma$, the integration over $E_{L}$ in Eqs. (A7) can be performed analytically using Eqs. (A4) for the amplitudes $b_{1,2}(t)$. As a result we finally arrive at Eq. (12) obtained from Eq. (7) for the case of non-interacting electrons. This agreement with the case of non-interacting electrons is quite remarkable since our rate equations dealing with many-electron states are very different from those obtained in the single electron framework. Yet, this is not surprising since in the case of non-interacting electrons the single electron description is valid. In fact, Eqs. (A3) can be mapped to Eq. (10) using $\left|\mathrm{b}_{i}(t)\right|^{2}=\sigma_{i i}(t)+$ $\sigma_{33}(t)$, where $i=1,2$ and $\mathbf{b}_{1}(t) \mathbf{b}_{2}^{*}(t)=\sigma_{12}(t)$. 


\section{REFERENCES}

[1] S.A. Gurvitz, IEEE Transactions on Nanotechnology 4, 45 (2005); (cond-mat/0406010).

[2] Y. Manassen, R.J. Hamers, J.E. Demuth, A.J. Castellano, Phys. Rev. Lett. 62, 2531 (1989); D. Shachal, Y. Manassen, Phys. Rev. B 46, 4795 (1992); Y. Manassen, I. Mukhopadhyay, N.R. Rao, Phys. Rev. B 61, 16223 (2000).

[3] C. Durkan, and M. E. Welland, Appl. Phys. Lett. 80, 458 (2002).

[4] D. Mozyrsky, L. Fedichkin, S.A. Gurvitz and G.P. Berman, Phys. Rev. B66, 161313(R) (2002).

[5] M. Braun, J.König and J. Martinek, Phys. Rev. B70, 195345 (2004); ibid, Superlattices and Microstructures 37, 333 (2005).

[6] S. Braig and P. W. Brouwer, Phys. Rev. B 71, 195324 (2005).

[7] J.N. Pedersen, J.Q. Thomassen, and K. Flensberg, Phys. Rev. B72, 045341 (2005).

[8] L. Y. Gorelik, S. I. Kulinich, R. I. Shekhter, M. Jonson, and V. M. Vinokur, Phys. Rev. Lett. 95, 116806 (2005).

[9] I. Weymann, J. König, J. Martinek, J. Barnas, and G. Schön, Phys. Rev. B72, 115334 (2005).

[10] Y. Chye, M.E. White, E. Johnston-Halperin, B.D. Gerardot, D.D. Awschalom, and P.M. Petroff, Phys. Rev. B66, 201301(R) (2002).

[11] M.M. Deshmukh and D.C. Ralph, Phys. Rev. Lett. 89, 266803 (2002).

[12] A. Jensen, J. R. Hauptmann, J. Nygard, and P.E. Lindelof, Phys. Rev. B72, 035419 (2005).

[13] A.N. Pasupathy, R.C. Bialczak, J. Martinek, J.E. Grose, L.A.K. Donev, P.L. McEuen, and D.C. Ralph, Science 306, 86 (2004). 
[14] S. Yuasa, T. Nagahama, A. Fukushima, Y. Suzuki, and K. Ando, Nature Materials 3, 868 (2004).

[15] S.A. Gurvitz and Ya.S. Prager, Phys. Rev. B53, 15932 (1996).

[16] S.A. Gurvitz, Phys. Rev. B57, 6602 (1998).

[17] J. Bardeen, Phys. Rev. Lett. 6 57, (1961); S.A. Gurvitz, "Two-potential approach to multi-dimensional tunneling", in Michael Marinov Memorial Volume, Multiple facets of quantization and supersymmetry, (Eds. M. Olshanetsky and A. Vainshtein, World Scientific), p. 91, (2002), (nucl-th/0111076).

[18] Note that in the large bias limit considered here, the off-diagonal matrix elements in the reservoir variables, $\sigma_{j j^{\prime}}^{n n^{\prime}, m m^{\prime}}$, are decoupled in the equation of motion from the diagonal elements, $\sigma_{j j^{\prime}}^{n n, m m} \equiv \sigma_{j j^{\prime}}^{n, m}$ (see also S.A. Gurvitz, Quantum Information Processing, 2, $15(2003))$.

[19] D.K.C. MacDonald, Rep. Prog. Phys. 12, 56 (1948).

[20] L.Y. Chen and C.S. Ting, Phys. Rev. B 43, R4534 (1991); ibid, 46, 4714 (1992); B. Elattari and S.A. Gurvitz, Phys. Lett. A 292, 289 (2002).

[21] Y.M. Blanter and M. Büttiker, Phys. Rep. 336, 1 (2000).

[22] R. Aguado and T. Brandes, Phys. Rev. Lett. 92, 206601 (2004).

[23] I. Bar-Joseph and S.A. Gurvitz, Phys. Rev. B44, 3332 (1991); S.A. Gurvitz, Phys. Rev, B44, 11924 (1991). 